\title{
Input and Output Connections of an Anteromedial Group of Spiking Local Interneurons in the Metathoracic Ganglion of the Locust
}

\author{
Toshiki Nagayama ${ }^{a}$ and Malcolm Burrows \\ Department of Zoology, University of Cambridge, Cambridge CB2 3EJ, England
}

Intracellular recordings were made from an anteromedial group of spiking local interneurons in the metathoracic ganglion of the locust to determine the input connections that shape their mechanoreceptive flelds on a hindleg and the output connections that define their effects on hindleg motor neurons.

The receptive fields of these interneurons may contain excitatory and inhibitory regions. An excitatory region on the ipsilateral hind leg is formed by direct excitatory connections of the afferents from exteroceptors. Afferent spikes consistently evoke EPSPs in interneurons with a central synaptic latency of 1.2-1.5 msec. The connections appear to be direct and chemically mediated.

An inhibitory region of a receptive field is not formed by direct afferent connections. Instead, spiking local interneurons of a midline group, which also receive direct excitatory inputs from the afferents, make direct inhibitory connections with anteromedial interneurons. Spikes in particular midline interneurons consistently evoke IPSPs in anteromedial interneurons with a central synaptic latency of about $1.0 \mathrm{msec}$. The connections appear to be direct and chemically mediated. By contrast, spikes in anteromedial interneurons could not be linked to any synaptic potentials in midline interneurons, so that direct connections are unidirectional and inhibitory.

The large inhibitory regions to the receptive fields of anteromedial interneurons result from convergent inhibitory inputs of several midline interneurons. Each midline interneuron may make inhibitory output connections with several anteromedial interneurons, but there is a high degree of specificity in these divergent connections.

Anteromedial interneurons make excitatory output connections with specific hind leg motor neurons. EPSPs in motor neurons follow spikes in the interneurons with short and consistent synaptic latencies of about 1.0 msec. The connections appear to be direct and chemically mediated. Two or more anteromedial interneurons with overlapping receptive fields may converge onto one motor neuron, and, in turn, one interneuron may make divergent connections with several motor neurons of the same pool.

\footnotetext{
Received June 29, 1989; revised Sept. 5, 1989; accepted Sept. 7, 1989.

This work was supported by grants from the SERC (UK) and by NIII grant NS 16058 to M.B. We thank our Cambridge colleagues for their helpful input to this work during the experimental stages and for their comments on the manuscript.

Correspondence should be addressed to M. Burrows, Department of Zoology, University of Cambridge, Downing Street, Cambridge CB2 3EJ, England.

a Present address: Zoological Institute, Faculty of Science, Hokkaido University, Sapporo 060, Japan.

Copyright (C) 1990 Society for Neuroscience $0270-6474 / 90 / 030785-10 \$ 02.00 / 0$
}

This pattern of connections suggests that these interneurons are essential elements in the local circuitry responsible for the expression of local reflexes of a leg. They provide an excitatory drive to the motor neurons in parallel to that from nonspiking local interneurons when particular arrays of exteroceptors are stimulated.

Local interneurons are essential elements in the transformation of mechanosensory signals received by receptors on a leg into appropriate reflex movements which adjust posture and locomotion (Siegler, 1984). The accessibility of these small interneurons in the locust for physiological, morphological, and ultrastructural study means that their integrative processes and the patterns of their connections with other neurons can be directly correlated with behavior. For example, touching an array of exteroceptors on a leg leads to a postural adjustment of that leg (Siegler and Burrows, 1986). The reflex movement, which involves the coordination of several joints of the leg, depends on the location of the receptors that are stimulated, so that the same stimulus delivered to different arrays of receptors leads to different but equally adaptive movements. The first step in understanding the control of such movements is to identify the neurons that are involved, describe their patterns of connections, and determine the interactions that create the complex motor patterns.

Many of the tactile exteroceptors on a locust leg are innervated by single sensory neurons which then make divergent excitatory synaptic connections with central neurons. Spiking local interneurons with cell bodics at the ventral midline of cach thoracic ganglion are a major recipient of these synaptic inputs (Siegler and Burrows, 1983). Divergent connections are, however, made with nonspiking local interneurons (Laurent and Burrows, 1988a), with intersegmental interneurons (Laurent and Burrows, 1988b), and with some motor neurons (Laurent and Hustert, 1988). The afferent signals are therefore processed in a parallel and distributed fashion by neurons with differing integrative properties. The dominant feature of this processing is that spatial information is preserved and that functional maps of the leg are constructed for the execution of specific patterns of movements.

The activation of an appropriate group of motor neurons results from the interactions between spiking and nonspiking local interneurons. Spiking local interneurons of the midline group make divergent inhibitory connections with nonspiking interneurons (Burrows, 1987), with intersegmental interneurons (Laurent, 1987), and with some motor neurons (Burrows and Siegler, 1982). The nonspiking interneurons make either excitatory or inhibitory connections with motor neurons (Burrows 
and Siegler, 1978) and inhibitory connections with other nonspiking interneurons (Burrows, 1979). The networks consist, therefore, of lateral inhibitory connections among the local interneurons and forward connections with the motor neurons and intersegmental interneurons. Inhibition predominates, to the extent that activation of the motor neurons appears to result mainly from disinhibition with a lesser contribution from direct excitatory afferent connections.

Within these networks, however, the action of a second group of spiking local interneurons has yet to be determined. These interneurons have cell bodies in an anterior and medial region of the ganglion and have extensive branches within half of the ganglion (Nagayama, 1989a). Most are affected by inputs from tactile receptors on the ipsilateral hind and middle legs, and some also by inputs from the contralateral hind and middle legs (Nagayama, 1989b). Each interneuron is excited or inhibited by a particular array of receptors that constitute the excitatory and inhibitory regions of its receptive field. They will therefore necessarily be affected by tactile stimuli that evoke local reflex movements of one leg (Siegler and Burrows, 1986) and by stimuli to other legs that modify the expression of these local reflexes (Laurent and Burrows, 1989). Here, we show the patterns of connections that underlie the action of these local interneurons. The excitatory regions of their receptive fields on the ipsilateral hind leg are formed by direct excitatory connections of afferents from exteroceptors, and the inhibitory regions by direct synaptic connections from spiking local interneurons of the midline group. In contrast to the midline spiking local interneurons, these interneurons make direct excitatory connections with hind leg motor neurons.

\section{Materials and Methods}

Adult locusts, Schistocerca gregaria (Forskål), of either sex were taken from our crowded laboratory culture and mounted ventral surface uppermost with all 6 tibiae and tarsi free to move. The meso- and metathoracic ganglia were stabilized on a wax-coated platform, and the thoracic cavity was perfused with a continuous flow of fresh saline throughout the experiments. The sheath of the metathoracic ganglion was treated with a $1 \%(\mathrm{wt} / \mathrm{vol})$ solution of protease (Sigma type XIV) in saline for 1-2 min before recording began.

Intracellular recordings, using microelectrodes filled with $2 \mathrm{M}$ potassium acetate, were made from the cell bodies of midline (Siegler and Burrows, 1984) and anteromedial (Nagayama, 1989a) spiking local interneurons, and from motor neurons that innervate muscles in a hind leg. Each local interneuron was initially located by the position of its cell body and then characterized physiologically according to its response to tactile stimulation of hairs (trichoid sensilla) on the surface of a hind leg. Individual hairs were moved manually with the aid of a fine artist's brush (Burrows and Siegler, 1985). The method allows the spatial domain of the stimulation to be controlled accurately. Temporal consistency of stimulation was achieved by always allowing at least 15 sec to elapse before a movement of a hair was repeated. To complete the identification, selected interneurons in each group were stained using electrodes filled with $0.1 \mathrm{M}$ hexamminecobaltic chloride (Brogan and Pitman, 1981). The detailed morphology of the interneurons is described elsewhere (Siegler and Burrows, 1984; Nagayama, 1989a). Interneurons with different receptive fields have different morphologies. No interneurons have yet been found that have the same receptive field but different morphologies. The motor neurons were identified by depolarizing individuals with current injected intracellularly and observing the movements of a hind leg. All recordings were stored on FM magnetic tape for later analysis and display. The results on the input connections are based on 40 paired recordings from midline and anteromedial spiking local interneurons. Connections were found between 5 pairs $(12.5 \%)$. The results on the output connections are based on 62 paired recordings from anteromedial interneurons and hind leg motor neurons in 40 locusts. Direct connections were found between 9 pairs (14.5\%).

\section{Results}

\section{Direct connections of afferents with interneurons}

To test whether the receptive fields of anteromedial interneurons on the ipsilateral hind leg are formed by direct connections of the hair afferents, intracellular recordings were made from these interneurons while recording the spikes of individual hair afferents extracellularly. Moving a single hair on the proximal tibia evokes spikes in its single afferent neuron and a depolarization and spikes in an anteromedial interneuron (Fig. 1A). Superimposed sweeps triggered from the afferent spikes show that EPSPs in the interneuron consistently follow each spike with a constant latency (Fig. $1 B$ ). In this example, there is a delay of $17.1 \mathrm{msec}$ between the afferent spike and the start of the EPSP in the interneuron because the afferent spikes are recorded $29.5 \mathrm{~mm}$ from the metathoracic ganglion. The afferent spikes have a conduction velocity of $1.9 \mathrm{~m} / \mathrm{sec}$ and within the ganglion would take at least $0.3 \mathrm{msec}$ to reach the synaptic sites. The synaptic delay is therefore about $1.3 \mathrm{msec}$. For 5 other interneurons tested in this way, the range of synaptic latencies is $1.2-1.5 \mathrm{msec}$, values similar to those measured for the connections with midline spiking local interneurons (Siegler and Burrows, 1983). Moreover, when a midline interneuron and an anteromedial interneuron are recorded simultaneously, the latency of the EPSPs evoked by electrical stimulation of afferent neurons is the same in both (Fig. 1C). The EPSPs can sum to produce spikes in the interneuron, and their amplitude is increased when the membrane of the interneuron is hyperpolarized with an applied current. These results are consistent with the connection being direct and chemically mediated.

\section{Direct inhibitory connections from midline spiking local interneurons}

The inhibitory fields on the ipsilateral hind leg do not, by contrast, result from direct inhibitory connections of the afferents with the interneurons. Spikes in the hair afferents could not be linked in a 1:1 fashion with IPSPs in anteromedial interneurons even when examined with signal averaging. Moreover, when the afferent nerve is stimulated electrically, an IPSP may be evoked in an anteromedial interneuron that occurs some $3 \mathrm{msec}$ later than an EPSP in a midline interneuron (Fig. 1D). An interposed interneuron is therefore most probably implicated in the inhibitory pathway to the anteromedial interneurons. The search for these interposed interneurons was concentrated upon spiking local interneurons in the midline group (Siegler and Burrows, 1984).

Mechanical stimulation of a specific array of hairs on a hind leg excites particular spiking local interneurons of the midline group and simultaneously inhibits particular interneurons of the anteromedial group (Fig. 2). In the example shown, touching a few hairs on the dorsal surface of the distal femur increases the frcquency of spikes in a midline interneuron and suppresses the spontaneous spikes of an anteromedial interneuron (Fig. 2A). To determine whether the spikes in the midline interneuron cause the inhibition in the anteromedial interneuron, a pulse of depolarizing current was injected intracellularly into the midline interneuron. The spikes in the midline interneuron evoked by this direct stimulation suppress the spontaneous spikes of the anteromedial interneuron and are associated with the occurrence of small hyperpolarizing synaptic potentials (Fig. $2 B$ ). On an expanded time scale, and with several traces superimposed, each spike in the midline interneuron is seen to be followed by 


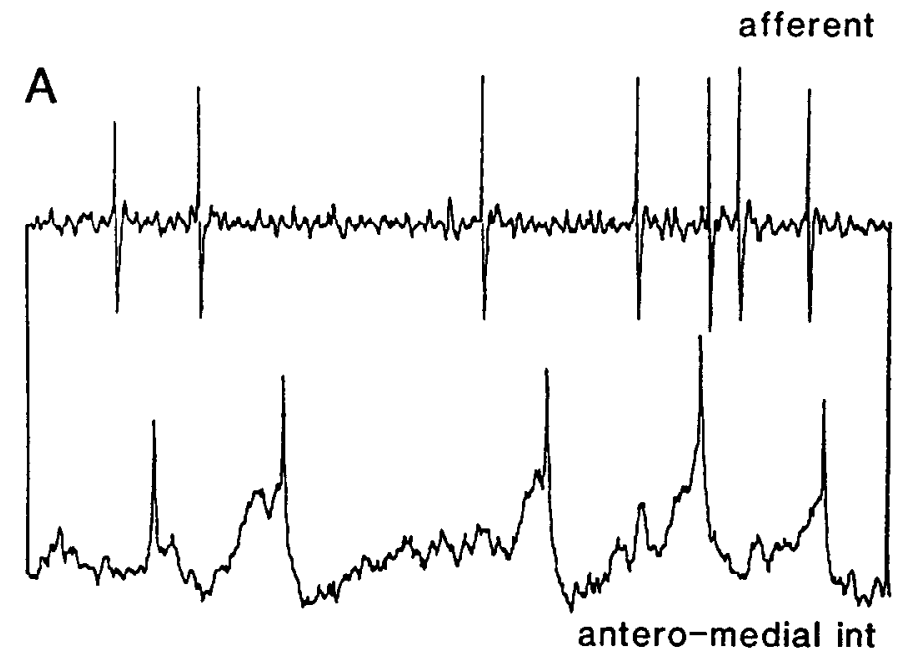

C

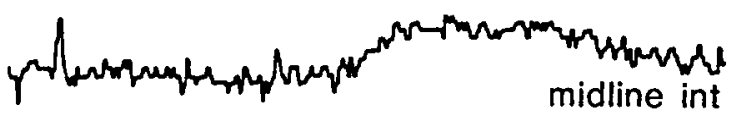

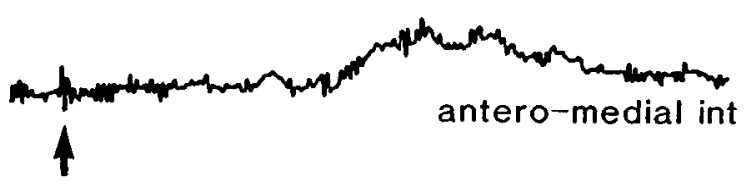

B

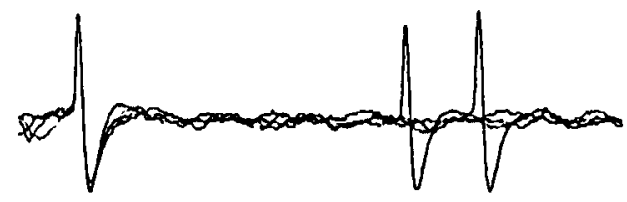
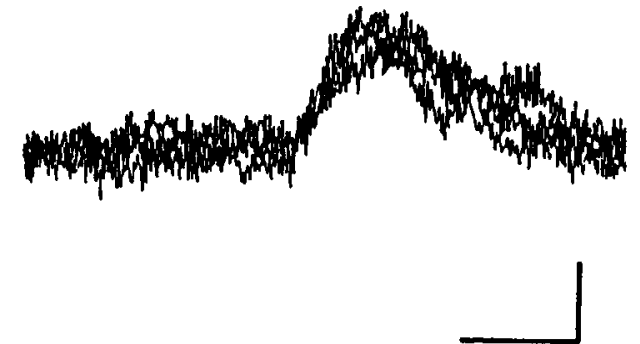

D

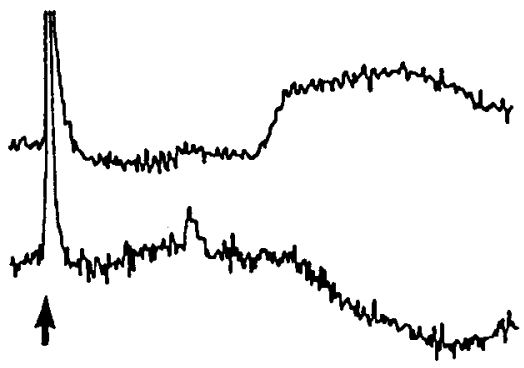

Figure 1. Hair afferents on a hind leg make direct excitatory connections with an anteromedial interneuron. $A$, Moving a hair on the proximal dorsal tibia evokes spikes in its afferent and a depolarization and sometimes a spike in an interneuron. $B$, Superimposed sweeps triggered from the afferent spikes show that EPSPs follow consistently and with a constant latency. $C$, Electrical stimulation of the afferent evokes EPSPs with the same latency in a midline and an anteromedial interneuron recorded simultaneously. Records are signal averages of 8 sweeps, triggered from the stimulus. $D$, Electrical stimulation of the afferent nerve evokes a short-latency EPSP in another midline interneuron and a longer-latency IPSP in another anteromedial interneuron. Records are signal averages of 32 sweeps. $A$ and $B$ are recordings from one locust, $C$ and $D$ from another. Calibration: voltage: $A, 5 \mathrm{mV} ; B, 2 \mathrm{mV}$; time: $A, 22 \mathrm{msec} ; B, 10 \mathrm{msec} ; C, 6.5 \mathrm{msec} ; D, 11 \mathrm{msec}$

a constant and short-latency hypcrpolarizing synaptic potential (IPSP) in the anteromedial interneuron (Fig. 2C). A steady depolarizing current injected into the anteromedial interneuron increases the amplitude of this IPSP, whereas a hyperpolarizing current decreases it (Fig. 2D). These results suggest, therefore, that the midline interneuron makes a chemically mediated, synaptic connection with the anteromedial interneuron.

The connections from midline interneurons to the anteromedial interneurons appear to be direct. In the 5 interconnected pairs of interneurons that were studied, the latencies, measured from the peak of the spikes to the start of the IPSPs, range from $0.9-1.1 \mathrm{msec}$ (see also Fig. 2, $C, D$ ). The connections are, however, one-way because spikes in anteromedial interneurons could not be correlated with synaptic potentials in midline interneurons even when signal averaging was used. The same result was obtained for all 40 pairs of interneurons recorded. Furthermore, a steady frequency of spikes in a midline interneuron is not altered when a high frequency of spikes is evoked in an anteromedial interneuron by the injection of a pulse of depolarizing current.

\section{Convergence of inputs from midline interneurons onto an anteromedial interneuron}

The inhibitory receptive fields of anteromedial interneurons is often large (Nagayama, 1989b). For example, the interneuron in Figure 3 has an inhibitory receptive field encompassing all of the hind leg that was accessible in this experiment. By contrast, no single midline interneuron has such an extensive excitatory receptive field (Burrows and Siegler, 1985). This suggests that the inhibitory receptive field of an anteromedial interneuron results from the convergent inhibitory inputs of several midline interneurons. To test this idea, 7 midline interneurons were sampled sequentially in one experiment while recording from one anteromedial interneuron (Figs. 3, 4),

The inhibitory receptive field of this anteromedial interneuron is formed by convergent inputs from at least 3 midline interneurons (Figs. 3, 4). The first midline interneuron is excited by mechanosensory inputs from the dorsal, distal femur (Fig. $3 A$ ) and from the ventral surface of both the tibia and tarsus (Fig. 3, $B, C$ ). It makes a direct inhibitory connection with the 
Figure 2. Inhibition of an anteromedial interneuron by a midline interneuron. $A$, Touching hairs on the dorsal surface of the distal femur (arrowhead) evokes a burst of spikes in the midline interneuron and inhibits the spikes in the anteromedial interneuron. $B, A$ pulse of depolarizing current injected into the midline interneuron through an unbalanced bridge circuit to evoke spikes inhibits the anteromedial interneuron. $C$, Multiple sweeps triggered by the spike of the midline interneuron show the constant latency of the IPSPs in the anteromedial interneuron. $D$, The amplitude of the IPSPs is increased by a steady depolarizing current and decreased by a hyperpolarizing current injected into the anteromedial interneuron. Each record is an average of 128 sweeps. Calibration: vertical voltage: $A$, $6 \mathrm{mV} ; B$, midline interneuron, $9 \mathrm{mV}$; anteromedial interneuron, $8.3 \mathrm{mV} ; C$, $1 \mathrm{mV}$; current: $1.3 \mathrm{nA}$; horizontal: $A$, $200 \mathrm{msec}, B, 360 \mathrm{msec}$; $C$ and $D, 10$ A antero-medial interneurone
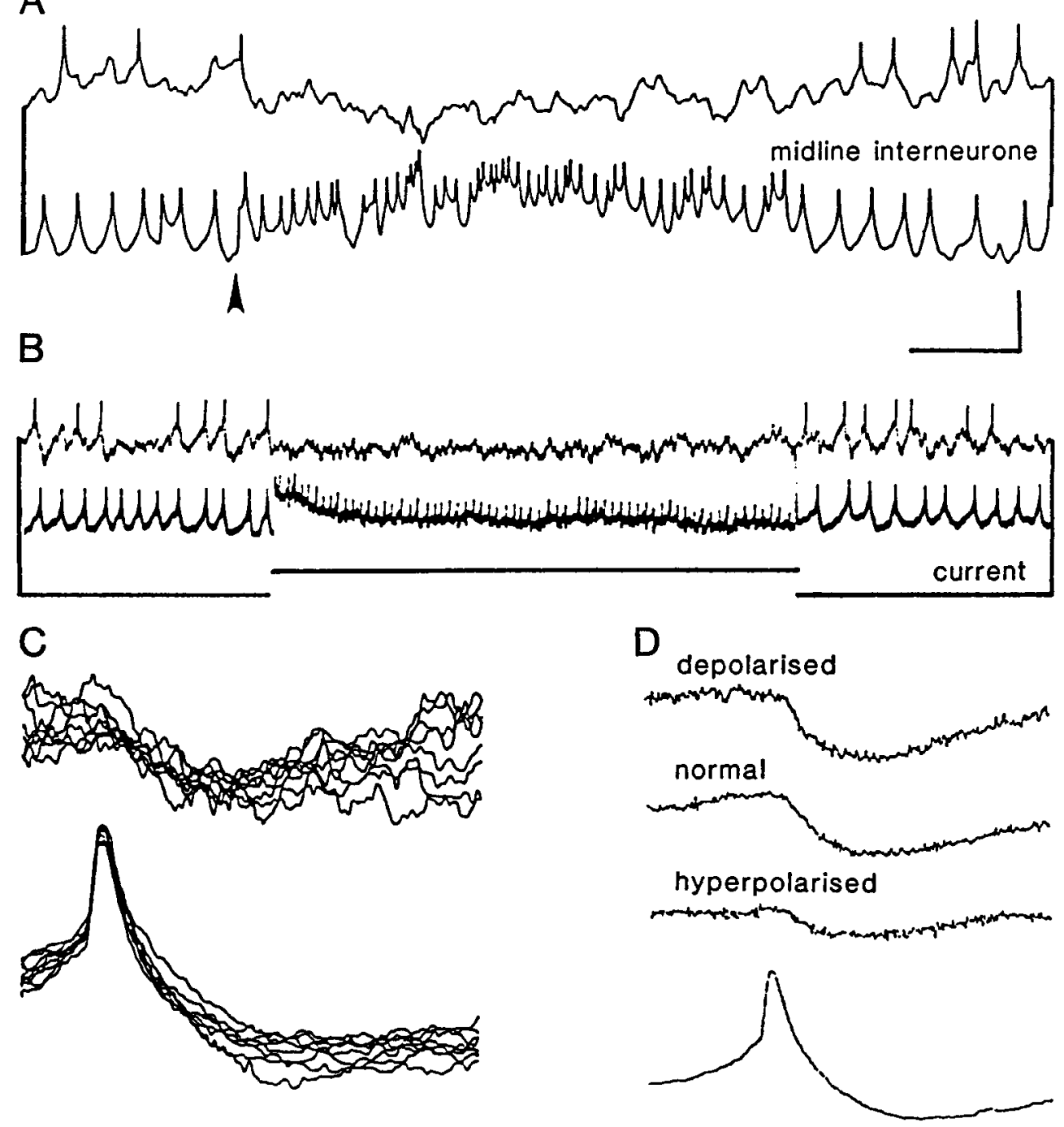
msec.

anteromedial interneuron (Fig. 3D). The second midline interneuron is excited by hairs on the whole of the ventral tibia (Fig. $3 E$ ) and on the dorsal, proximal tibia (Fig. $3 F$ ). It also makes a direct inhibitory connection with the anteromedial interneuron (Fig. 3G). The third midline interneuron is excited by hairs on the dorsal, distal femur, the dorsal tibia, and the ventral, distal tibia (Fig. 4). It also makes a direct inhibitory connection with the anteromedial interneuron. The remaining 4 midline interneurons are excited by hairs that are within the receptive field of the anteromedial interneuron, but none make inhibitory connections with it (Fig. 4). These results indicate that overlap in the receptive fields of pairs of interneurons is not a reliable indicator of connectivity.

\section{Divergence of connections made by a midline spiking local interneuron}

Midline spiking local interneurons make divergent output connections with several anteromedial interneurons. For example, the midline interneuron shown in Figure 5 synapses with at least 3 anteromedial interneurons. Connections with the first anteromedial interneuron are shown in Figures 3 and 4. Touching hairs on the distal, dorsal femur excites the midline interneuron and inhibits the second anteromedial interneuron (Fig. 5A). Inputs from the distal, dorsal femur (Fig. $5 B$ ), and from the ventral tibia and the ventral tarsus excite the midline interneuron and inhibit the third anteromedial interneuron. Signal averages triggered by spikes in the midline interneuron show that IPSPs follow at a constant and short latency in the anteromedial interneurons (Fig. 5, C, D). The similar latency from the peak of a spike in the midline interneuron to the start of an IPSP in all 3 anteromedial interneurons suggests that the connections are direct.

\section{Direct excitatory connections with leg motor neurons}

The search for possible output connections of anteromedial interneurons was concentrated upon the motor neurons that are responsible for movements of the tarsus. Touching a few hairs on the ventral surface of the tibia evokes an increase in the frequency of spikes in an anteromedial interneuron and a depolarization of a fast depressor tarsi motor neuron (Fig. 6A). If the same stimulus is repeated with the interneuron hyperpolarized, the number of its spikes is reduced and the depolarization of the motor neuron is correspondingly reduced (Fig. 6, $B, C$ ). This suggests that the tactile stimulus activates several pathways 
A

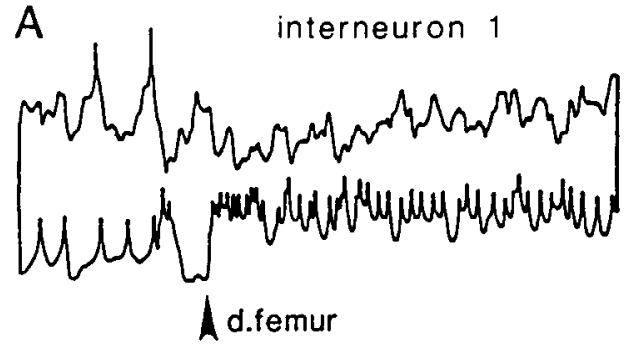

B

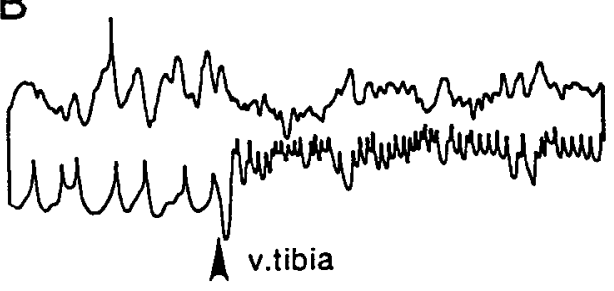

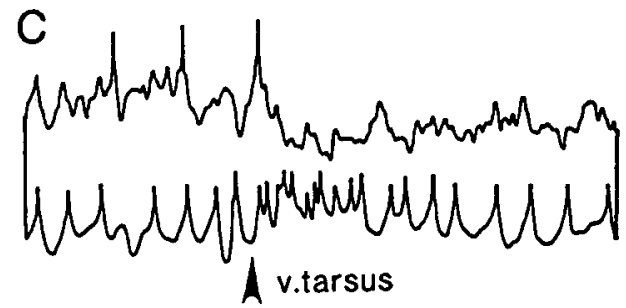

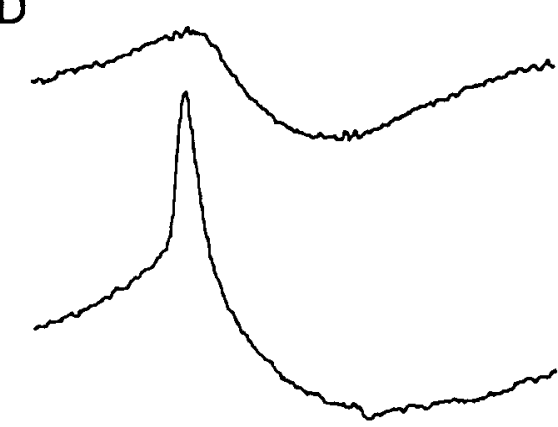

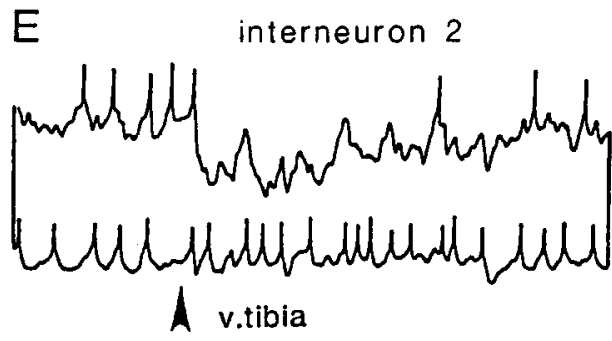

F

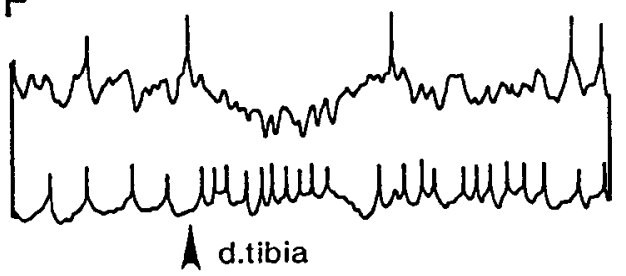

G
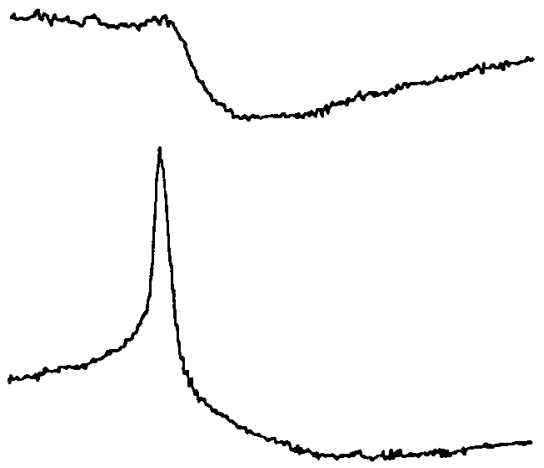

Figure 3. Convergence of inputs from 2 midline interneurons onto an anteromedial interneuron. $A-C$, Touching hairs (arrowheads) on the dorsal distal femur $(A)$, the ventral proximal tibia $(B)$, and the ventral tarsus $(C)$ excites the first midline interneuron and inhibits the anteromedial interneuron. $D$, A signal-averaged record (256 sweeps) triggered from spikes in the midline interneuron shows a direct inhibitory connection with the anteromedial interneuron. $E$ and $F$, Touching hairs on the ventral $(E)$ or dorsal tibia $(F)$ excites the second midline interneuron and inhibits the anteromedial interneuron. $G$, A signal-averaged record (256 sweeps) shows a direct inhibitory connection. Calibration: vertical: $A-C$, midline interneuron, $10 \mathrm{mV}$, anteromedial interneuron, $3 \mathrm{mV} ; E$ and $F$, both interneurons, $9.6 \mathrm{mV}$; horizontal: $A-C, E$, and $F, 300 \mathrm{msec} ; D$ and $G, 9 \mathrm{msec}$. which converge on the motor neuron, one of which involves the anteromedial interneuron. When a pulse of depolarizing current is injected directly into the anteromedial interneuron to evoke spikes, the membrane potential of the motor neuron is again depolarized by a barrage of EPSPs, some of which can be correlated with the spikes in the interneuron (Fig. 6D). When more current is injected, the motor neuron is gradually depolarized and then more abruptly produces a larger depolarization and subsequent oscillations in its membrane potential (Fig. $6 E$ ). This suggests that the spikes in the anteromedial interneuron also activate other motor neurons that cause movements of the leg. The depolarization of the impaled depressor tarsi motor neuron thus results from the combined effects of central pathways and peripheral feedback from the movement.

Spikes in this anteromedial interneuron are always followed by small depolarizing EPSPs in the depressor tarsi motor neuron (Fig. 7A). The superimposed sweeps show the consistency of the relationship between the spikes and the EPSPs, and the constancy of the latency at 1.0 msec (Fig. $7, A, B$ ). In 8 other pairs of interneurons and tarsal motor neurons the latency ranged from 1.0-1.3 msec. The EPSPs may sum to produce a sustained depolarization of the membrane and evoke motor spikes, but usually they can only reliably be revealed by signal averaging (Fig. 7B). A steady hyperpolarizing current injected into the motor neuron increases the amplitude of the EPSPs (Fig. 7B). These results indicate that the EPSPs of the motor neuron are evoked by chemically mediated, direct connections from the anteromedial interneuron.

This anteromedial interneuron also synapses on a slow depressor tarsi motor neuron (Fig. 7, C, D). A pulse of depolarizing current injected into the interneuron increases the frequency of spikes in the motor neuron (Fig. 7C). Signal averages triggered by the spikes in the interneuron show that the EPSPs in the slow depressor motor neuron occur with the same latency as that recorded for the fast depressor motor neuron (Fig. $7 D$ ). Thus, a single anteromedial interneuron makes divergent connections with at least 2 motor neurons of the same pool.

The outputs of several interneurons converge onto the same 
Figure 4. Specificity of connections made by midline interneurons. The receptive fields of 7 midline interneurons containing regions common to the anteromedial interneuron described in Figure 3 are shown. Midline interneurons 1-3 make direct inhibitory synapses with the anteromedial interneuron but interneurons 4-7 do not. Midline interneuron 1 is described in Figure 3. In the diagrams of receptive fields, the surface of hind leg is represented as if the femur, tibia, and tarsus are extended fully, and opened by an incision along the ventral midline. Excitatory regions are crosshatched, inhibitory ones are stippled.
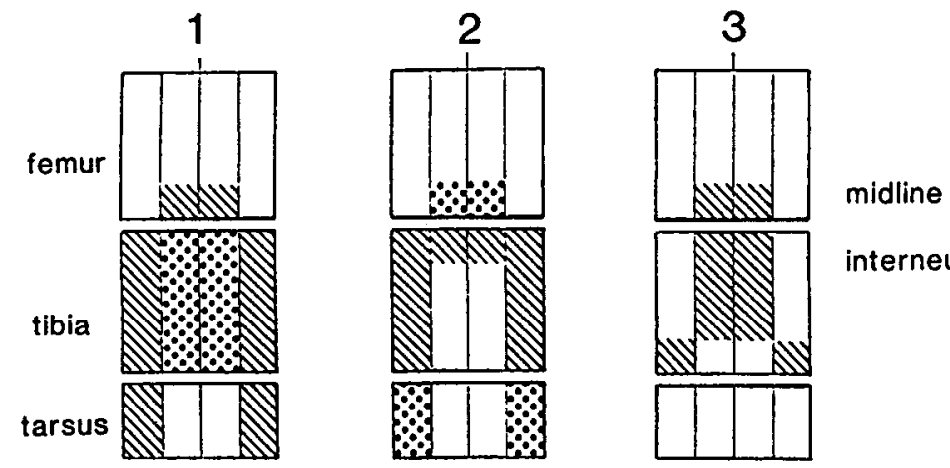

interneurones antero-medial

interneurone
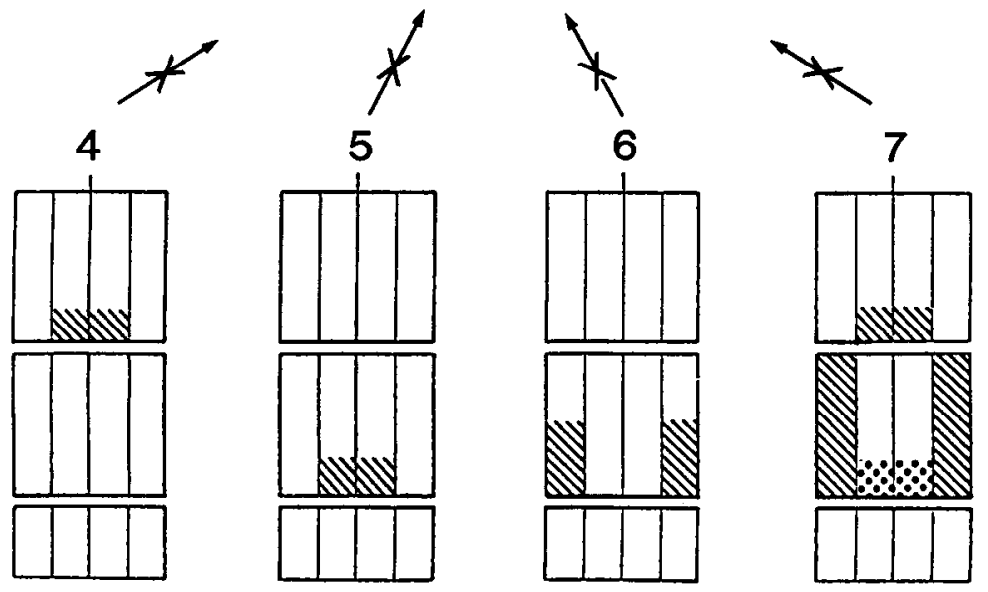

midline interneurones

motor neuron (Fig. 8). Two interneurons which receive excitatory inputs from dorsal tibial hairs make direct connections with a depressor tarsi motor neuron (Fig. 8, $A, B$ ). These interneurons and the one in Figure 7 therefore provide a convergent input to this motor neuron in response to tactile stimulation of the dorsal tibia. In total, 6 different anteromedial interneurons were found to make direct and excitatory connections with depressor tarsi motor neurons. No direct inhibitory connections with leg motor neurons have been found.

\section{Discussion}

Connections of the anteromedial interneurons

The intracellular recordings show that spiking local interneurons in an anteromedial group receive excitatory inputs from affer- ents of exteroceptors on a hind leg and inhibitory inputs from specific spiking local interneurons of the midline group. These connections shape the receptive field of an interneuron. In turn, the anteromedial interneurons make excitatory output connections with motor neurons that innervate muscles of the hind leg from which they receive the excitatory afferent input. These 3 sets of effects all appear to be caused by direct, chemically mediated synaptic connections. The synaptic potentials consistently follow the presynaptic spikes with a latency of about 1 msec, and their amplitude depends on the membrane potential of the postsynaptic neuron. The central delays are similar to those measured for connections between other neurons in the locust CNS that are thought to be monosynaptic. For example, the central latencies for the connections of hair afferents with 


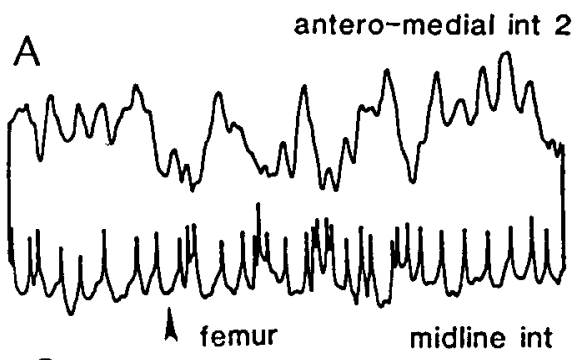

C

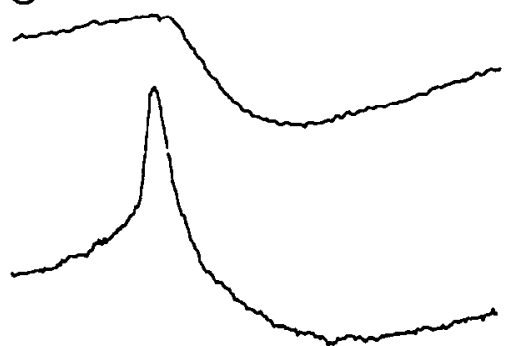

E
$\mathrm{B}$

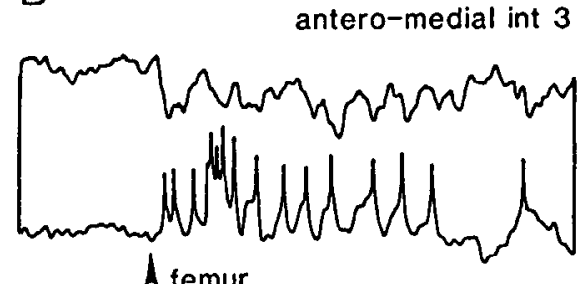

D midline int

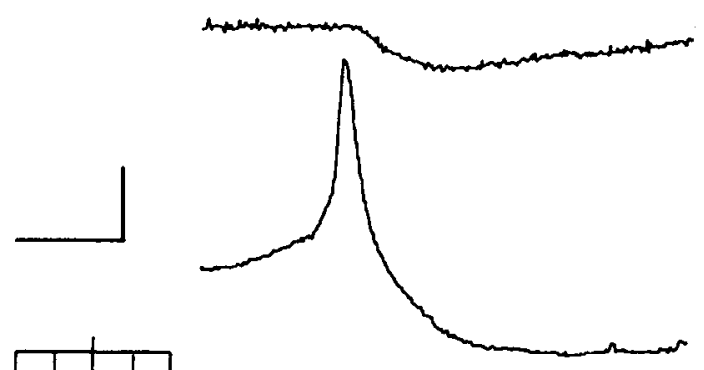

midline

interneurone

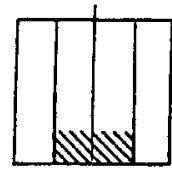

Figure 5. Divergence of connections from a midline interneuron to 3 anteromedial interneurons. $A$ and $B$, Touching hairs on the dorsal distal femur (arrowheads) excites the midline interneuron and inhibits anteromedial interneurons 2 and $3 . C$ and $D$. Signal averages (256 sweeps) triggered from spikes in the midline interneuron show a linked IPSP with the same latency in both anteromedial interneurons. $E$, Diagram to show the receptive fields of the interneurons and the connections revealed. The inhibitory connection from the midline interneuron to the first anteromedial interneuron is shown in Figure $3 A$. Calibration: vertical: anteromedial interneuron in $A, 8 \mathrm{mV} ; B, 5$ $\mathrm{mV}$; midline interneuron in $A, 4 \mathrm{mV}$; $B, 6 \mathrm{mV}$; horizontal: $A$ and $B, 200 \mathrm{msec}$;

antero-medial interneurones

spiking local interneurons of the midline group (Siegler and Burrows, 1983) are similar and, moreover, simultaneous recordings from a midline and an anteromedial interneuron show that EPSPs occur at the same time in both.

The anatomy of the neurons involved in these pathways is consistent with this interpretation of the physiological results. The afferents from the hairs project to the most ventral areas of neuropil (Pflüger et al., 1981), while the branches of leg motor neurons project to more dorsal and lateral areas (Watkins et al., 1985). Both the midline and anteromedial interneurons have distinctive layers of branches (Siegler and Burrows, 1984; Nagayama, 1989a), one of which overlaps the terminal branches of the hair afferents and the other the branches of the motor neurons (Siegler and Burrows, 1984; Nagayama, 1989a).

\section{Construction of receptive fields}

The excitatory regions to the receptive fields of anteromedial interneurons on a hind leg are formed by the direct connections of afferents from hairs. Each afferent connects with more than one interneuron so that spatial information about a region of the leg is distributed among several members of the population. By contrast, the inhibitory regions of the receptive fields are formed by the connections made by midline spiking local interneurons which receive direct excitatory inputs from arrays 

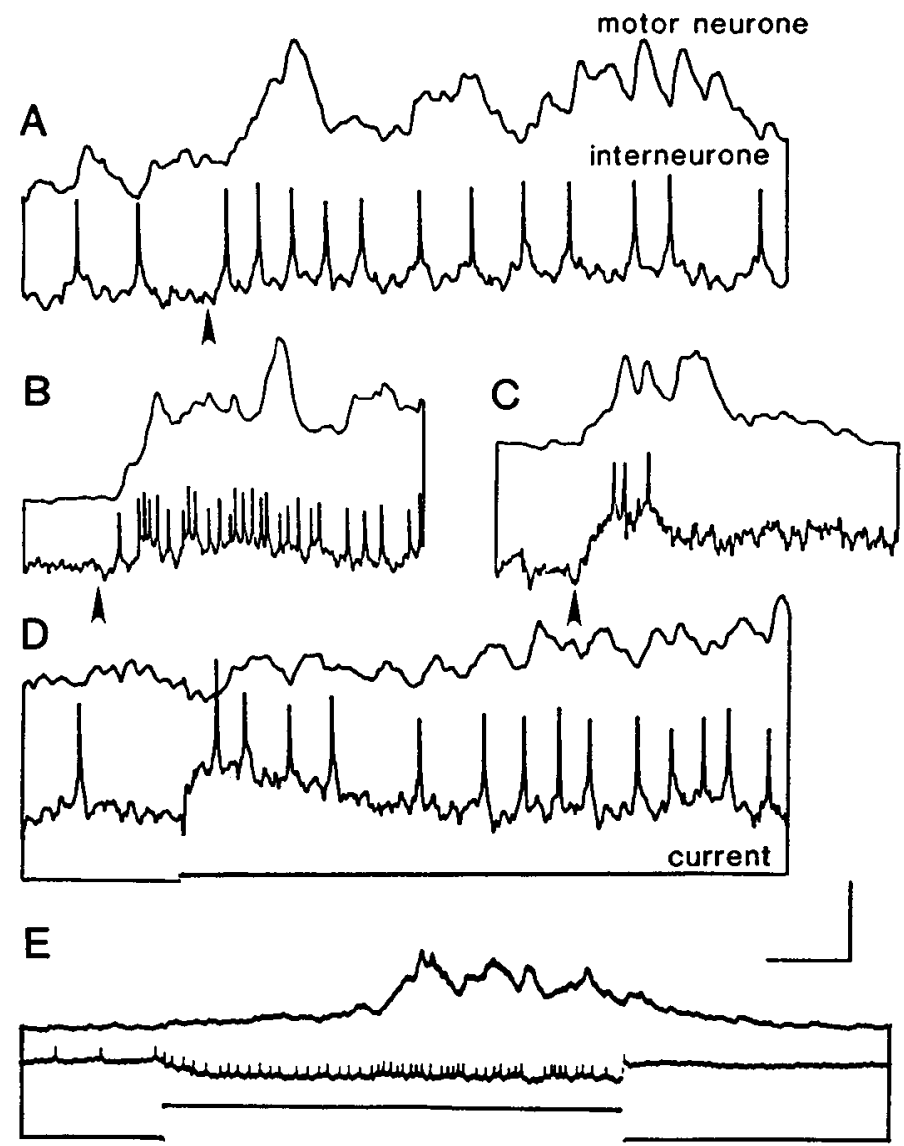

Figure 6. Excitation of a hind leg motor neuron by an anteromedial spiking interneuron. $A$ and $B$, Touching hairs on the ventral surface of the proximal tibia (arrowhead) evokes a burst of spikes in the interneuron and a depolarization in a fast depressor tarsi motor neuron. $C$, The tactile stimulus is repeated while the interneuron is held hyperpolarized by a steady current. The response in the motor neuron is reduced. $D$, Spikes are evoked in the interneuron by a pulse of depolarizing current, and the motor neuron is depolarized by series of depolarizing potentials that are associated with each spike. $E, A$ larger current is injected into the interneuron, and the motor neuron at first depolarizes slowly but then more abruptly. Calibration: vertical: $A$ and $D$, motor neuron, $8 \mathrm{mV}$; interneuron, $4 \mathrm{mV} ; B$ and $C$, motor ncuron, $6 \mathrm{mV}$; interneuron, $6.4 \mathrm{mV} ; E$, motor neuron, $34 \mathrm{mV}$; interneuron, 27 $\mathrm{mV}$; current, $6 \mathrm{nA}$; horizontal: $A$ and $D, 67 \mathrm{msec} ; B$ and $C, 200 \mathrm{msec}$; $E, 240 \mathrm{msec}$.

of receptors and then reverse their sign. Moreover, anteromedial interneurons with purely inhibitory receptive fields have restricted ventral branches and none in the most ventral regions of the neuropil (Nagayama, 1989b), where the terminal branches of hair afferents from the hind leg project (Pflüger et al., 1981).
These connections mean that part of the inhibitory field of an anteromedial interneuron will correspond to the excitatory field of a particular midline interneuron. Many anteromedial interneurons, however, have larger inhibitory fields than the excitatory field of an individual midline interneuron. These large fields result from convergent inhibitory inputs of several midline interneurons, often with parts of their excitatory fields in common. Furthermore, one midline interneuron makes divergent inhibitory connections with several anteromedial interneurons, but not every midline interneuron with a region of its receptive ficld in common with that of an anteromedial intcrncuron forms connections. Nevertheless, each anteromedial interneuron has a characteristic receptive field even though some regions are shared by other interneurons in the group (Nagayama, 1989a). The uniqueness of a receptive field is therefore determined by serial and parallel processing through the highly ordered connections of afferents and midline interneurons.

\section{Control of motor output}

The anteromedial interneurons make excitatory connections with hind leg motor neurons in contrast to the inhibitory connections made by midline spiking local interneurons (Burrows and Siegler, 1982; Burrows, 1987; Laurent, 1987). Immunocytochemical evidence shows that none of the anteromedial interneurons have GABA-like immunoreactivity, whereas many midline interneurons do (Watson, 1986; Watson and Burrows, 1987). Particular anteromedial interncurons conncet with several members of a motor neuron pool, and in turn, each motor neuron is excited by several interneurons. The connections are, however, highly specific because not all interneurons with an excitatory input from one particular region of the hind leg synapse with the same motor neuron. Not all of the observed effects on the leg motor neurons are explained by these connections and more complex pathways are implicated. These may involve parallel pathways within the ganglion and the excitation of other motor neurons causing movements of the leg that generate sensory feedback.

\section{Organization of local reflex pathways}

Tactile stimulation of exteroceptors on a hind leg leads to specific reflex movements that depend on the location of the receptors (Siegler and Burrows, 1986). The anteromedial interneurons must now be added to the pathways that organize these movements. Consider an array of receptors on one part of the hind leg that when stimulated leads to a particular movement and an array elsewhere that leads to a complementary movement (Fig. 9). In the first movement a particular motor neuron is excited and in the second it is inhibited. To excite the motor neuron the afferents may synapse directly on it (Laurent and Iustert, 1988) and excite parallel pathways that involve non-

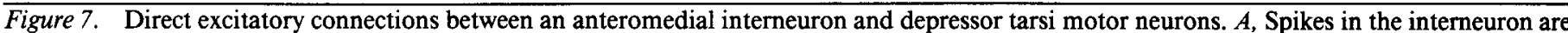

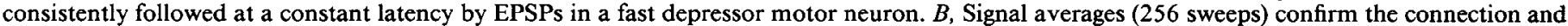

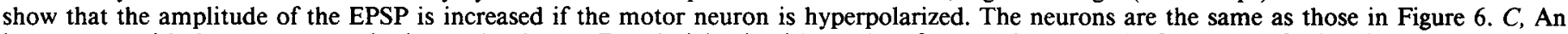

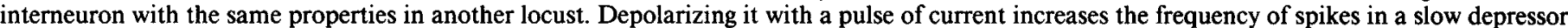

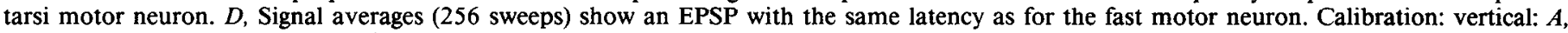
$1.5 \mathrm{mV}$; $C$, motor neuron, $8 \mathrm{mV}$; interneuron, $10 \mathrm{mV}$; current, $10 \mathrm{nA}$; horizontal: $A, B$, and $D, 5 \mathrm{msec} ; C, 250 \mathrm{msec}$.

Figure 8. Convergence of inputs from 2 anteromedial interneurons onto a leg motor neuron. $A$ and $B$, Multiple sweeps triggered by the spike of the first $(A)$ or second $(B)$ interneuron show constant and short-latency EPSPs in a fast depressor tarsi motor neuron. The diagrams show the receptive fields of the interneurons. The interneuron in Figure $7 A$ also converges on this motor neuron. Calibration: vertical: $A$, motor neuron, 1.2 $\mathrm{mV}$; interneuron, $4.8 \mathrm{mV}$; $B$, motor neuron, $0.5 \mathrm{mV}$; interneuron, $2 \mathrm{mV}$; horizontal: $10 \mathrm{msec}$. 


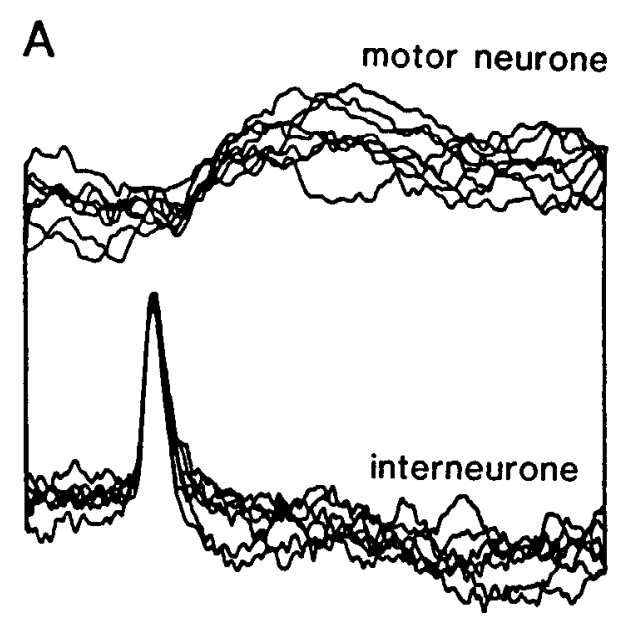

C

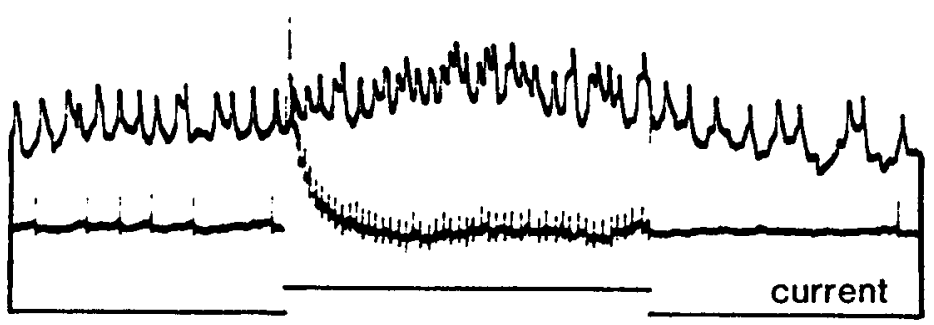

B
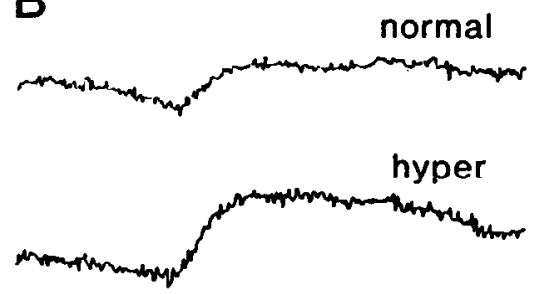

$-$

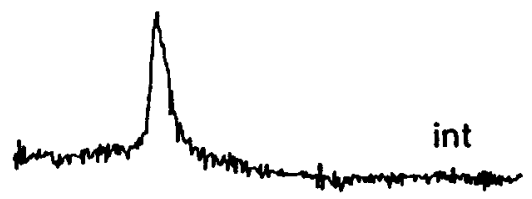

D

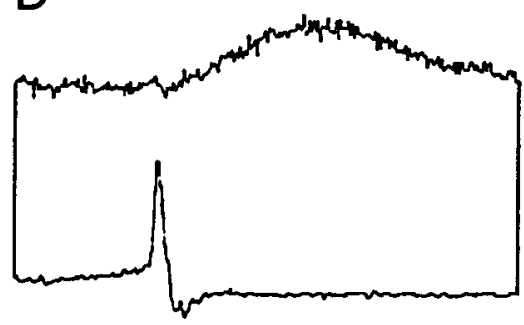

A

interneurone

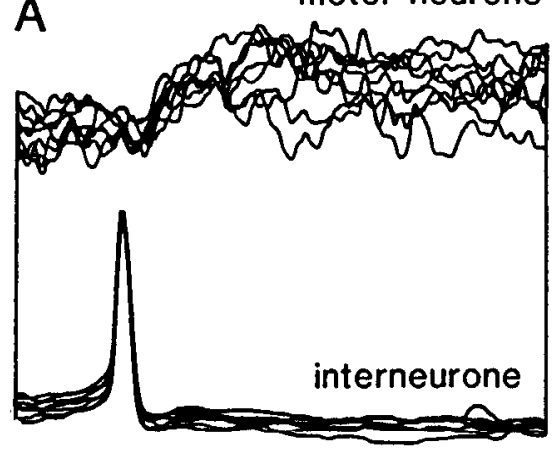

B

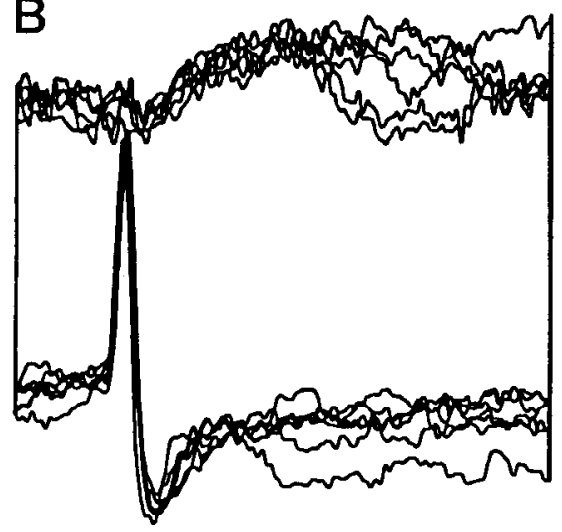

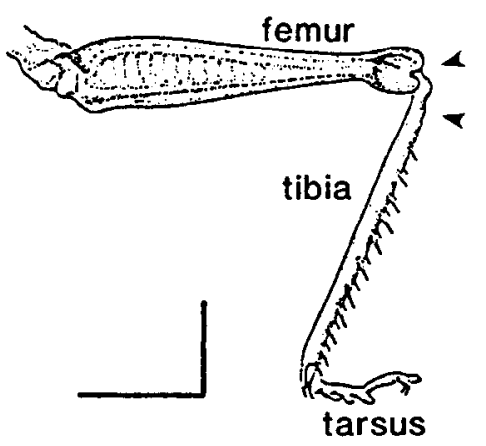
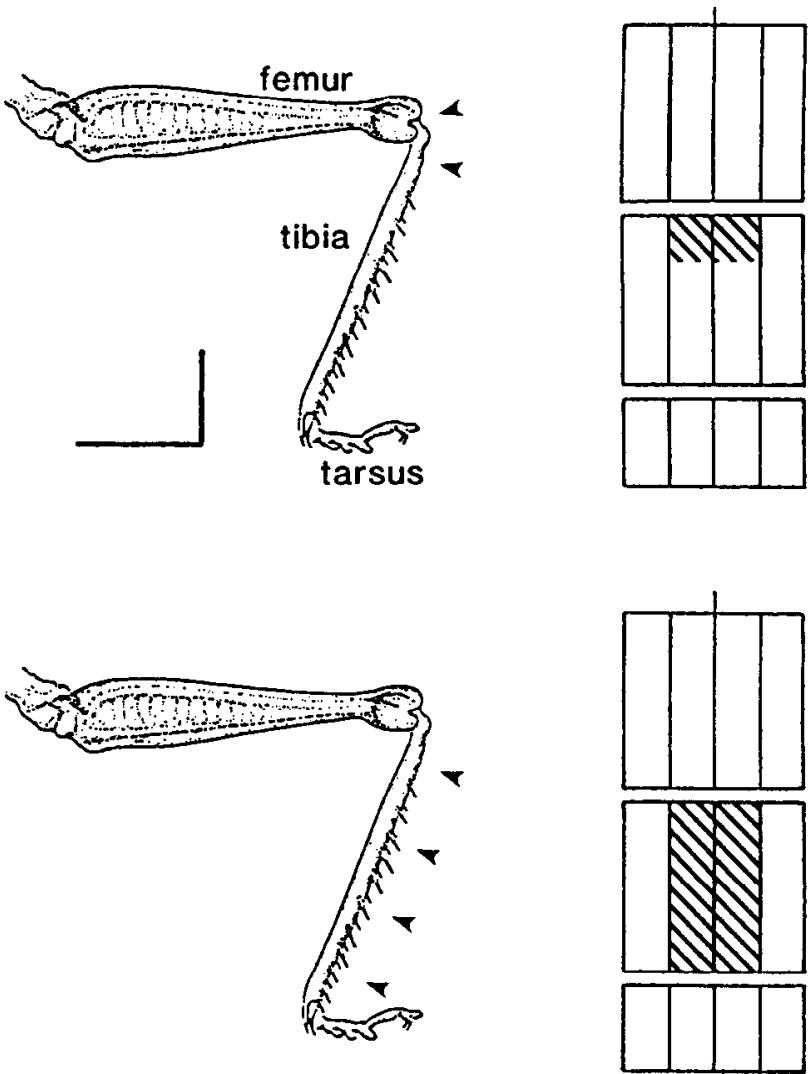


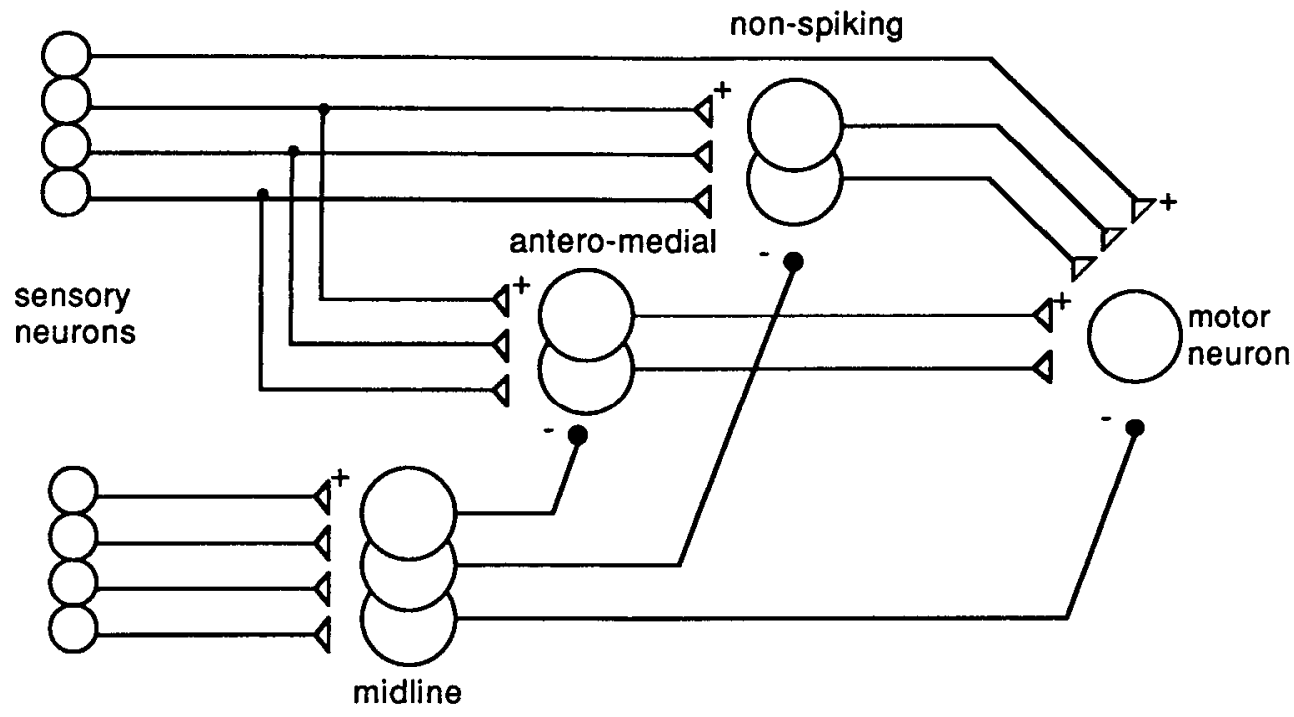

Figure 9. Diagram of the pathways involved in local reflexes of a hind leg. The 2 groups of afferent neurons are from arrays of receptors on different positions on the ipsilateral hind leg. The filled circles represent inhibitory synapses; open triangles, excitatory synapses. A full description is given in the text.

Burrows, M., and M. V. S. Siegler (1985) The organization of receptive fields of spiking local interneurones in the locust with inputs from hair afferents. J. Neurophysiol. 53: 1147-1157.

spiking local interneurons (Laurent and Burrows, 1988a) and anteromedial interneurons with excitatory output connections. Finally, disinhibition may play a part. Some nonspiking interneurons make 1-way inhibitory connections with other nonspiking interneurons (Burrows, 1979). Therefore, afferent excitation of a nonspiking interneuron that inhibits another nonspiking interneuron with inhibitory output connections will result in disinhibition of the motor neuron.

To inhibit the motor neuron the second array of afferents must act through the midline spiking local interneurons (Fig. 9). These interneurons make inhibitory synapses directly on the motor neuron (Burrows and Siegler, 1982), on nonspiking interneurons (Burrows, 1987), and on anteromedial interneurons with excitatory connections to the motor neuron. The effect of these last 2 sets of connections of the midline interneurons will be to suppress the excitatory effects of the other local interneurons.

Thus, the parallel and cascade connections through the anteromedial interneurons are another essential element in the control of leg movements in the locust. One effect is to transfer excitatory inputs from the afferents to particular leg motor neurons. A second is to bias the action of other local interneurons in the pathways. Moreover, since the receptive fields of many anteromedial interneurons include regions on other legs $(\mathrm{Na}$ gayama, 1989b), they could gate specific reflexes of a hind leg in accordance with movements of the other legs. This role would therefore be complementary to the gain control action of the nonspiking local interneurons (Laurent and Burrows, 1989).

\section{References}

Brogan, R. T., and R. M. Pitman (1981) Axonal regeneration in an identified insect motoneurone. J. Physiol. (Lond.) 319: 34-35P.

Burrows, M. (1979) Graded synaptic transmission between local premotor interneurons of the locust. J. Neurophysiol. 42: 1108-1123.

Burrows, M. (1987) Inhibitory interactions between spiking and nonspiking local interneurones in the locust. J. Neurosci. 7: 3282-3292.

Burrows, M., and M. V.S. Siegler (1978) Graded synaptic transmission between local interneurones and motoneurones in the metathoracic ganglion of the locust. J. Physiol. (Lond.) 285: 231-255.

Burrows, M., and M. V. S. Siegler (1982) Spiking local interneurons mediate local reflexes. Science (N.Y.) 217: 650-652.
Laurent, G. J. (1987) The role of spiking local interneurones in shaping the receptive fields of intersegmental interneurones in the locust. J. Neurosci. 7: 2977-2989.

Laurent, G. J., and M. Burrows (1988a) Direct excitation of nonspiking local interneurones by exteroceptors underlies tactile reflexcs in the locust. J. Comp. Physiol. A 162: 563-572.

Laurent, G. J., and M. Burrows (1988b) A population of ascending intersegmental interneurones in the locust with mechanosensory inputs from a hind leg. J. Comp. Neurol. 275: 1-12.

Laurent, G. J., and M. Burrows (1989) Intersegmental interneurones can control the gain of reflexes in adjacent segments by their action on nonspiking local interneurones. J. Neurosci. 19: 3030-3039.

Laurent, G. J., and R. Hustert (1988) Motor neuronal receptive fields delimit patterns of activity during locomotion of the locust. J. Neurosci. 8: 4349-4366.

Nagayama, T. (1989a) Morphology of a new population of spiking local interneurone in the locust metathoracic ganglion. J. Comp. Neurol. 283: 189-211.

Nagayama, T. (1989b) The organisation of receptive fields of an antero-medial group of spiking local interneurones in the locust with exteroceptive inputs from the legs. J. Comp. Physiol. (in press).

Pflüger, H. J., P. Bräunig, and R. Hustert (1981) Distribution and specific central projections of mechanoreceptors in the thorax and proximal leg joints of locusts. II. The external mechanoreceptors: Hair plates and tactile hairs. Cell Tissue Res. 216: 79-96.

Siegler, M. V. S. (1984) Local interneurones and local interactions in arthropods. J. Exp. Biol. 112: 253-281.

Siegler, M. V. S., and M. Burrows (1983) Spiking local interneurons as primary integrators of mechanosensory information in the locust. J. Neurophysiol. 50:1281-1295.

Siegler, M. V. S., and M. Burrows (1984) The morphology of two groups of spiking local interneurones in the metathoracic ganglion of the locust. J. Comp. Neurol. 224: 463-482.

Siegler, M. V. S., and M. Burrows (1986) Receptive fields of motor neurons underlying local tactile reflexes in the locust. J. Neurosci. 6 : 507-513.

Watkins, B. L., M. Burrows, and M. V. S. Siegler (1985) The structure of locust non-spiking interneurones in relation to the anatomy of their segmental ganglion. J. Comp. Neurol. 240: 233-255.

Watson, A. H. D. (1986) The distribution of GABA-like immunoreactivity in the thoracic nervous system of the locust Schistocerca gregaria. Cell Tissue Res. 246: 331-341.

Watson, A. H. D., and M. Burrows (1987) Immunocytochemical and pharmacological evidence for GABAergic spiking local interneurones in the locust. J. Neurosci. 7: 1741-1751. 